\title{
Regional improvement of global reanalyses by means of a new long-term Mediterranean hindcasted precipitation dataset: a first study over the Iberian Peninsula
}

\author{
M. G. Sotillo ${ }^{1}$, M. L. Martín ${ }^{2}$, F. Valero ${ }^{3}$, and M. Y. Luna ${ }^{4}$ \\ ${ }^{1}$ Área de Medio Físico, Ente Público Puertos del Estado (EPPE), Madrid, Spain \\ ${ }^{2}$ Escuela de Informática, Campus de Segovia, Universidad de Valladolid, Spain \\ ${ }^{3}$ Facultad de CC. Físicas, Universidad Complutense de Madrid, Spain \\ ${ }^{4}$ Instituto Nacional de Meteorología, Madrid, Spain
}

Received: 7 October 2005 - Revised: 1 December 2005 - Accepted: 19 December 2005 - Published: 19 April 2006

\begin{abstract}
Generation of a Mediterranean long-term (19582001) homogeneous high resolution environmental database constituted the main objective whitin the HIPOCAS Project. The high number of parameters included in this database allows a complete characterization of Mediterranean storms. In this paper, the HIPOCAS precipitation reliability over the Iberian Peninsula and the Balearic Islands is evaluated against long-term in-situ observations from Iberia. In order to provide a more complete study, comparisons of the HIPOCAS field with NCEP/NCAR and ERA global reanalysis show the important improvement in the characterisation of the observed precipitation introduced by the HIPOCAS hindcast.
\end{abstract}

\section{Introduction}

In general terms, the major methodological drawback for a long-term assessment of regional climate and its variability comes from the lack of suitable observation or simulated data. It is well known that the coarse spatial resolution of global reanalysis make these data sets a not completely adequate tool to characterize regional prevailing atmospheric conditions over areas marked by complex orography and land-sea distributions such as the Mediterranean Basin. In that sense, the generation within the HIPOCAS Project framework of a Mediterranean long-term (1958-2001) homogeneous high resolution atmospheric database comes to overcome the above-referred shortcomings providing a useful data set for regional studies. The present contribution is concerning to the HIPOCAS precipitation over the Iberian

Correspondence to: M. G. Sotillo

(marcos@puertos.es)
Peninsula and the Balearic Islands, evaluating its reliability against long-term in-situ observations from Iberia. Furthermore, the HIPOCAS significant improvement in comparison to NCEP/NCAR and ERA global reanalysis is evaluated.

\section{Data and methodology}

The HIPOCAS long-term database is the result of an atmospheric hindcast performed over the whole Mediterranean Basin. In order to produce the 44-year (1958-2001) hindcast the regional atmospheric model REMO was used. The whole Mediterranean basin was covered by a grid with horizontal resolution of $0.5^{\circ} \times 0.5^{\circ}$ (roughly $50 \times 50 \mathrm{~km}^{2}$ ). NCEP/NCAR global reanalysis (Kalnay et al., 1996) were used as initial and boundary conditions. An spectral nudging technique was applied to keep the regional run close to the imposed time-variable large-scale atmospheric states provided by the NCEP forcing. Further information on the HIPOCAS Mediterranean data base and its generation can be found in Sotillo et al. (2005).

A high-resolution daily precipitation data base derived from in-situ measurements coming from more than 4000 stations of the Spanish Meteorological Service (Instituto Nacional de Meteorología, INM) have been used to validate the HIPOCAS precipitation. Further information about the observed precipitation data set can be found in Luna and Almarza (2004). In order to validate the HIPOCAS hindcasted precipitation, 41-yr (1961-2001) winter observed Iberian monthly Precipitation data set (hereafter, IPD) was used. To this aim, the original daily data was interpolated to the HIPOCAS grid and monthly-accumulated precipitation values obtained. Additionally, monthly precipitation from NCEP/NCAR (Kalnay et al., 1996) and ERA (Gibson et al., 1997) global reanalyses were also interpolated from their 

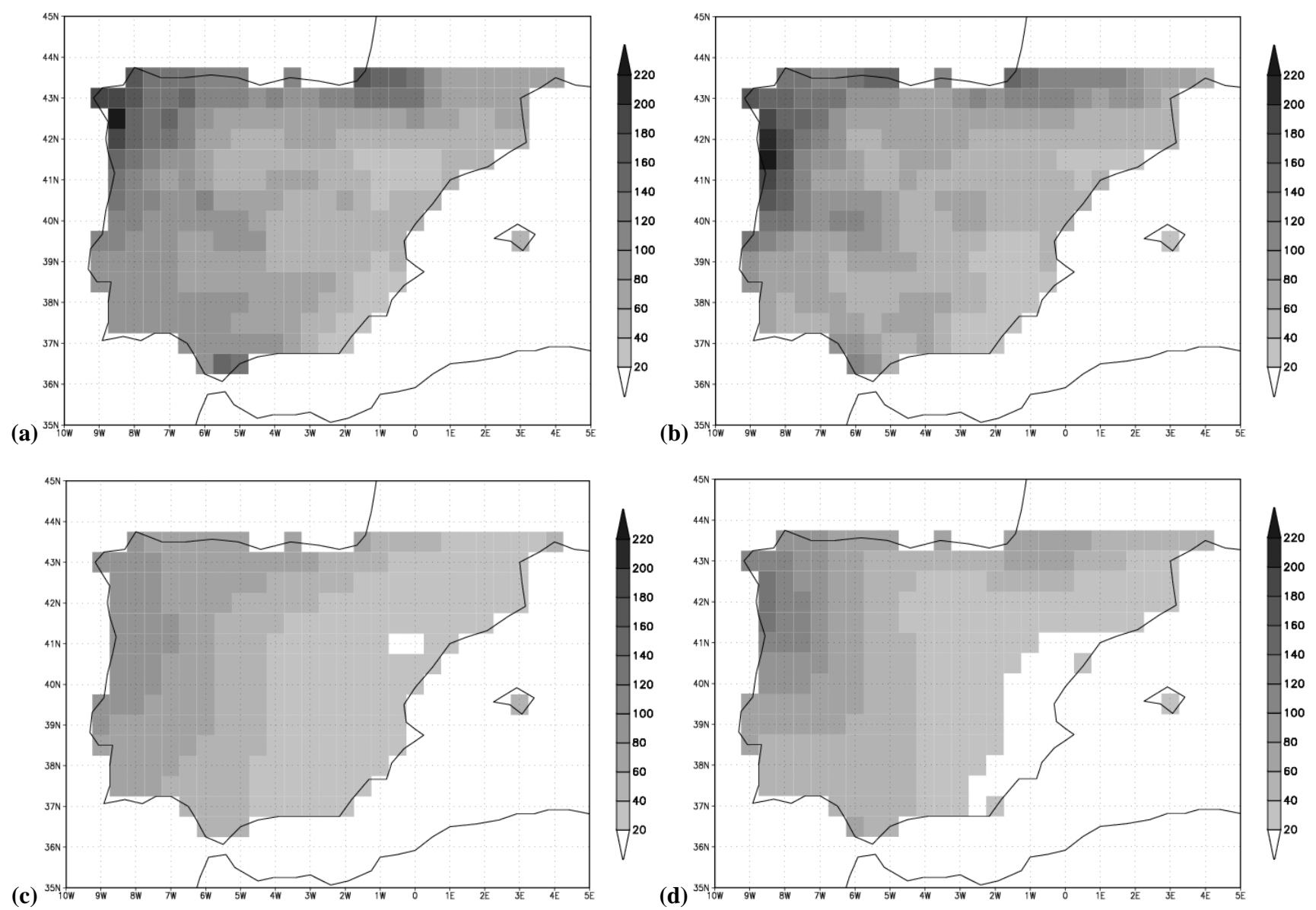

Fig. 1. Winter spatial distributions of monthly mean precipitation fields (mm) of: (a) IPD; (b) HIPOCAS; (c) NCEP and (d) ERA.

original resolution (around $200 \mathrm{~km}$ and $125 \mathrm{~km}$, respectively) to the HIPOCAS grid and were used in order to complete the HIPOCAS validation.

In order to validate the HIPOCAS winter precipitation dataset several products were derived. A general description of HIPOCAS, NCEP, ERA and IPD datasets was made by means of statistics such as precipitation means and root mean squared error (RMSE). The temporal evolution of the spatial average bias and RMSE were derived to evaluate the different model performance ability. Likewise, a principal component analysis (PCA) with a varimax orthogonal rotation procedure was applied to the databases in order to evaluate the different model performance ability in reproducing the observed spatial precipitation patterns, as well as their temporal evolution.

\section{Results}

This validation was performed through comparisons with the observed IPD set taking into account the described methodology. The 41-winter monthly mean IPD precipitation field (Fig. 1a) shows the well-known differences in precipitation behaviour between the Atlantic and Mediterranean areas of the Iberian Peninsula. The analogous HIPOCAS precipitation field (Fig. 1b) shows well agreement, not only in the spatial gradient but also in the absolute precipitation values. Similar comparison was performed using the NCEP and ERA global reanalysis data sets (Figs. 1c and d), showing similar spatial distributions with the maximum clearly lower than the observed IPD and hindcasted HIPOCAS. The RMSE spatial distributions (Figs. $2 b$ and c) show slight enhancement of ERA versus NCEP which is largely improved by the HIPOCAS performance (Fig. 2a).

From the rotated PCA to IPD and HIPOCAS fields, five rotated PCs, explained $90.4 \%$ of the total variance, of both fields were selected. Comparisons of simulated (Fig. 3) and observed (not shown) patterns illustrate how the HIPOCAS hindcasted field largely captures the main characteristics of the IPD field. The HIPOCAS patterns reproduce reasonably accurately observed regional characteristics linked to the main orographic features in the study domain. High time correlation values obtained between both PC time series point out high degree of similarity between them corroborating the model performance ability. Spatial distribution for time correlations between the observed and the HIPOCAS 

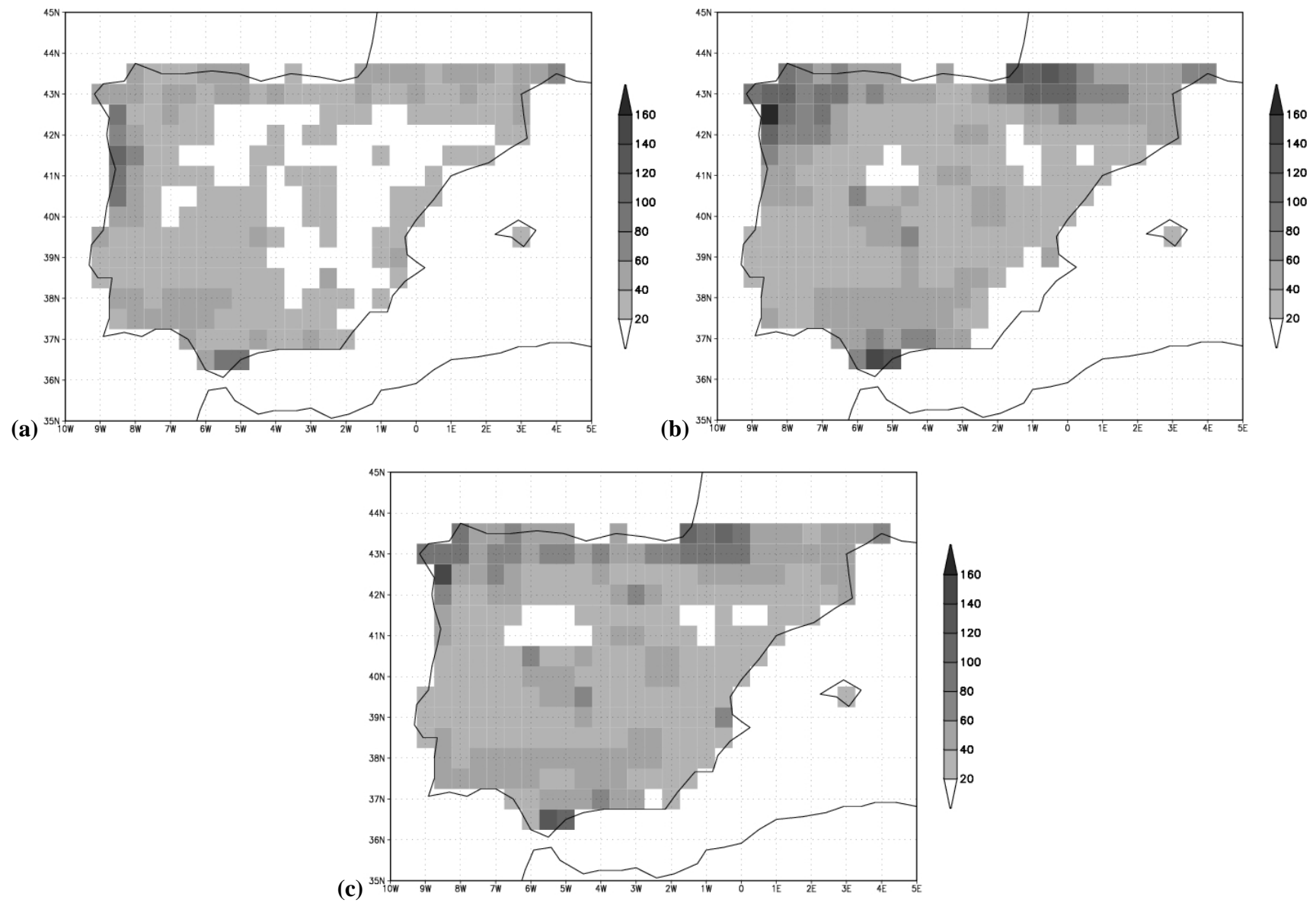

Fig. 2. Root mean squared error (mm) between (a) IPD and HIPOCAS fields; (b) IPD and NCEP fields and (c) IPD and ERA fields.

precipitation fields (Fig. 4) highlights strong agreement between both fields showing a clear E-W gradient with an increased westward correlation. Whereas the highest values (higher than 0.80) are located over the western side of the Iberian Peninsula, the lowest ones are located along the Mediterranean coast, specially in its southeastern part. This area shows low winter precipitation records (as can be seen in Fig. 1a), and it is characterized by a seasonal maximum in autumn, being this maximum linked to strong convective storm activity related to the typical intense Mediterranean ciclogenesis (Valero et al., 2004). It is also worth to mention that other HIPOCAS validation works focused on variables such as the 10-m wind field, waves and sea level data, have also identified the southeastern Iberia as an area where the HIPOCAS hindcast does not present its better performance (Sotillo et al., 2005).

In order to provide a more complete view of the NCEP, ERA and HIPOCAS performances that allow the observed Iberian precipitation field along the 41-year period to be characterised, Fig. 4 also displays the time evolution of the bias (Fig. 5a) and the RMSE (Fig. 5b) averaged over the whole spatial domain. Both figures highlight the much better HIPOCAS performance for reproducing the observed IPD field, in comparison with the global reanalysis data.

\section{Conclusions}

A general description of the Iberian winter precipitation reproduced by the Mediterranean HIPOCAS data base is shown. The HIPOCAS performance is validated through comparisons between such hindcasted data and the observed IPD observed precipitation fields, highlighting the existence of a very good agreement in terms of not only spatial and time distribution, but also in terms of total amount of precipitation. The important improvement in the characterisation of the observed precipitation introduced by the HIPOCAS hindcast in relation to the global reanalyses is also highlighted. It is worth to finally note that the strength in terms of confidence on the HIPOCAS precipitation data set resulting from the performed validation over Iberia along with the proved remarkable improvement in regards with the global reanalysis data sets, allow to recommend its use in regional Mediterranean climate studies. Furthermore, its use may be even helpful in climatological studies focused on specific areas, such as the offshore ones, handicapped by the lack of observations. 

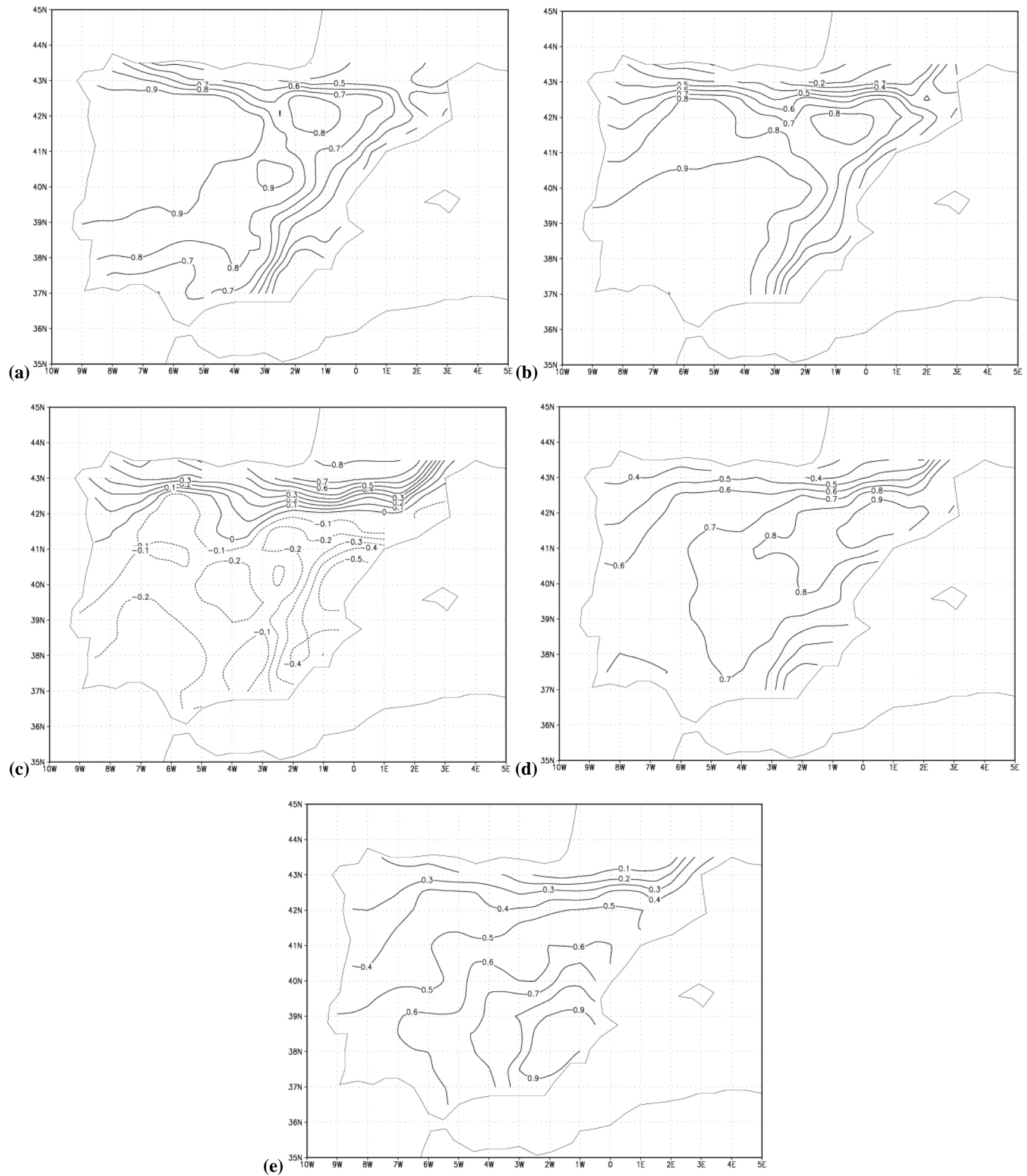

Fig. 3. Patterns of the selected Varimax rotated PCs corresponding to the HIPOCAS precipitation field: (a) first to (e) five. 


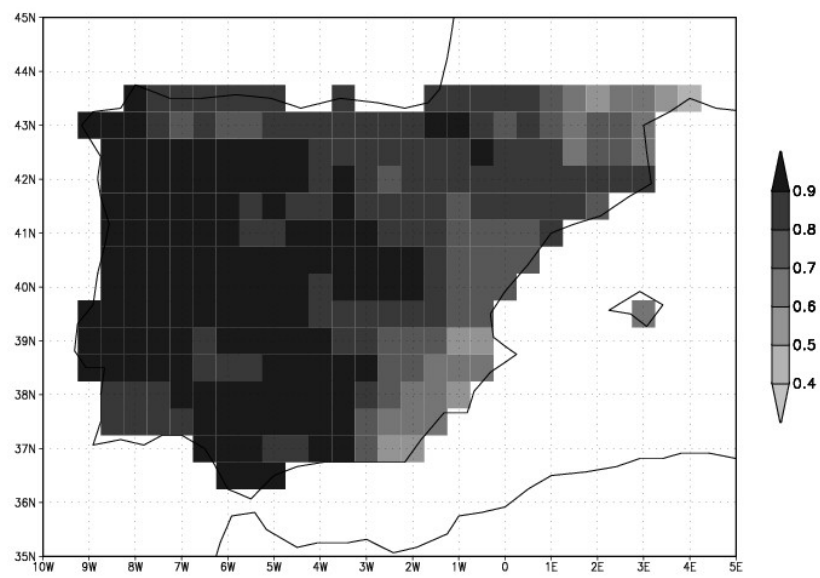

Fig. 4. Temporal correlation between IPD and HIPOCAS precipitation fields.

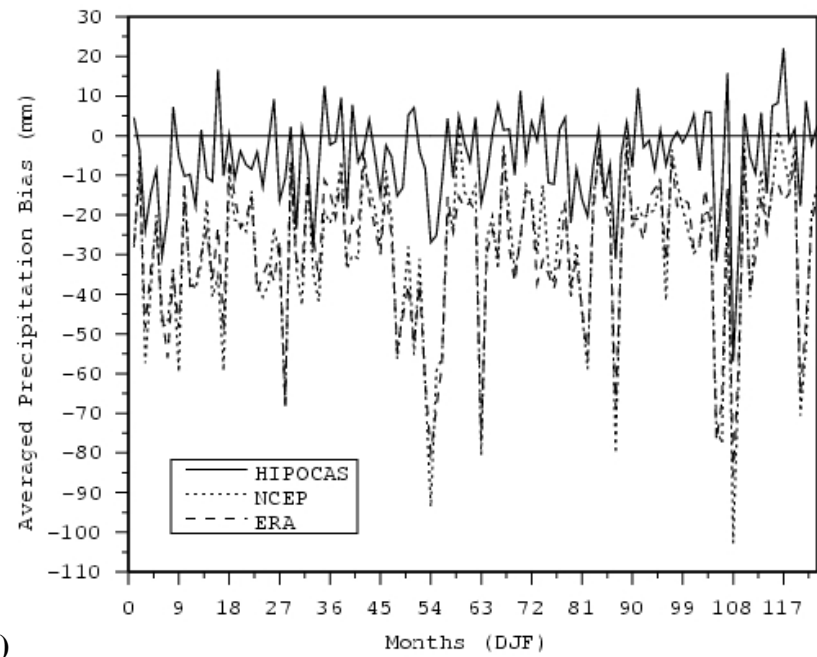

(a)

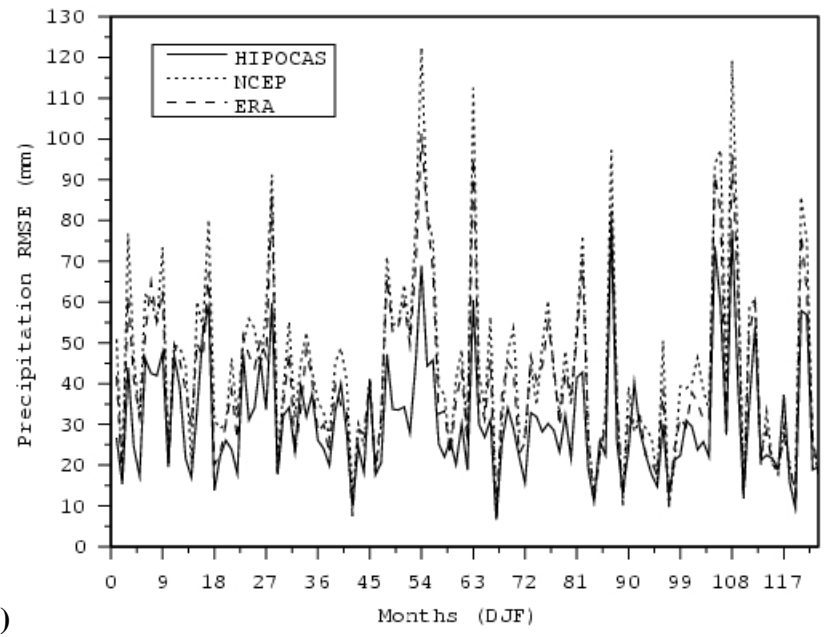

Fig. 5. Temporal evolution of: (a) the bias and (b) the root mean squared error averaged over the whole spatial domain for the used datasets.
Acknowledgements. This work has been partially supported by the research project CGL2004-01584/CLI. The authors wish to thank the following institutions which provided us with data: HIPOCAS data from Ente Público Puertos del Estado (EPPE, Spain), IPD from Spanish Meteorological Service (INM, Spain), NCEP Reanalysis data by the NOAA-CIRES Climate Diagnostics Center, Boulder, Colorado, USA and ERA40 from the European Centre for Weather Medium Forecast (ECWMF). The authors would like to thank the reviewers for their helpful comments which have contributed to improving this paper.

Edited by: V. Kotroni and K. Lagouvardos

Reviewed by: S. Lykoudis

\section{References}

Gibson, J. K., Kallberg, P., Uppala, S., Hernández, A., Ñomura, A., and Serrano, E. : ERA description. Technical Report Re-analysis Project Report Series 1, ECMWF, Reading, UK, 1997.

Kalnay, E., and co-authors: The NCEP/NCAR 40-years Reanalysis, Project. Bull. Amer. Meteorol. Soc., 77, 437-471, 1996.

Luna, M. Y. and Almarza, C.: Interpolation of 1961-2002 daily climatic data in Spain, Proc. International Meeting on Spatial Interpolation in Climatology and Meteorology, Budapest, Hungry, 2004.

Sotillo, M. G., Ratsimandresy, A. W., Carretero, J. C., Bentamy, A., Valero, F., González-Rouco, J. F.: A high-resolution 44-year atmospheric hindcast for the Mediterranean Basin: Contribution to the regional improvement of global reanalysis, Clim. Dyn., 25, 219-236, 2005.

Valero, F., Luna, M. Y., Martin, M. L., Morata, A., and GonzálezRouco F.: Coupled modes of large-scale climatic variables and regional precipitation in the Western Mediterranean in autumn, Clim. Dyn., 22, 307-323, 2004. 